\title{
Estudio del estado de conservación de las yeserías del Oratorio de la Madraza de Granada. Identificación, evaluación y análisis
}

\author{
Study of the conservation status of the plasterwork of the Oratory of the \\ Madrasah of Granada. Identification, evaluation and analysis
}

J. Ramos Molina ${ }^{(*)}$, J. A. Durán Suárez ${ }^{(* *)}$, E. Sebastián Pardo ${ }^{(* *)}$, M. $^{\text {a P. Sáez Pérez }}{ }^{(* * *)}$

\section{RESUMEN}

La realización de estudios sobre el estado de conservación previo a las intervenciones rehabilitadoras y restauradoras permite conocer y actuar de forma coherente sobre el patrimonio arquitectónico. En esta línea se ha llevado a cabo la investigación de localización gráfica de alteraciones, estudio puntual de materiales de alteración y medición de parámetros ambientales (T y H) en las yeserías del Oratorio de la Madraza de Granada, espacio singular por sus antecedentes históricos y constructivos.

Los resultados obtenidos determinan el nivel de alteración y presencia de los diferentes deterioros, así como la justificación de las causas de alteración que los han motivado. Igualmente se valida la metodología seguida en este estudio, consistente en diversos protocolos sucesivos que facilitan la determinación de las intervenciones restauradoras. De igual manera este método puede ser extrapolable a otros edificios con alto nivel de protección patrimonial.

Palabras clave: Madraza; Granada; Oratorio; yeserías; conservación; diagnosis.

\section{ABSTRACT}

State of conservation studies carried out prior to rehabilitation and restorative interventions allow to act consistently on architectural heritage. This has been done in this research, graphic location of alterations, study of materials for alteration and measuring of environmental parameters ( $T$ and $H$ ) in the plasterwork of the Oratory of the Madrasah of Granada, singular space by their constructive and historical background.

The results obtained determine the level of alteration and presence of different damages, as well as the justification of the causes of alteration to them have motivated. The methodology followed in this study, consisting of various successive protocols that facilitate the determination of restorative interventions is equally valid. This method can be extrapolated to other buildings with high level of protection heritage.

Keywords: Madraza; Granada; Oratory; plasterwork; conservation; diagnosis.

(*) Julia Ramos Restauración del Patrimonio, S. L.

(**) Departamento de Escultura. Universidad de Granada. (Granada, España).

(***) Departamento Mineralogía y Petrología. Universidad de Granada. (Granada, España).

(****) Departamento Construcciones Arquitectónicas. Universidad de Granada. (Granada, España).

Persona de contacto/Corresponding author: mpsaez@ugr.es (M. ${ }^{\text {P P. Sáez Pérez) }}$

ORCID: http://orcid.org/oooo-0001-5047-697X (J. Ramos Molina); http://orcid.org/oooo-0002-9714-3555 (J. A. Durán

Suárez); http://orcid.org/oooo-0002-0671-4309 (E. Sebastián Pardo); http://orcid.org/oooo-0001-9725-1153 (M. ${ }^{\text {a P. Sáez }}$ Pérez)

Cómo citar este artículo/Citation: Ramos Molina, J., Durán Suárez, J. A., Sebastián Pardo, E., Sáez Pérez, M. ${ }^{a}$ P. (2016). Estudio del estado de conservación de las yeserías del Oratorio de la Madraza de Granada. Identificación, evaluación y análisis. Informes de la Construcción, 69(545): e175, doi: http://dx.doi.org/10.3989/ic.16.088.

Copyright: (c) 2017 CSIC. Licencia / License: Salvo indicación contraria, todos los contenidos de la edición electrónica de Informes de la Construcción se distribuyen bajo una licencia de uso y distribución Creative Commons Attribution License (CC BY) Spain 3.o. 


\section{INTRODUCCIÓN}

El edificio conocido como La Madraza se considera de especial interés en la arquitectura nazarí. Toma su nombre del término «Madrasa», que en árabe significa escuela (1). Su origen se remonta a la Granada nazarí, fundada en el año 1349 bajo el reinado de Yusuf I (1333-1354), y por ello también fue llamada Madraza Yusufiyya.

Como centro de estudios superiores se dedicó a la enseñanza de las ciencias islámicas, particularmente la jurisprudencia y la teología, así como a la lengua y la literatura árabe, y otras ciencias como la medicina, la astronomía, la gramática, la lógica, la aritmética y la geometría (2). Fue la primera fundación docente en España de carácter oficial/estatal y la segunda en Al-Ándalus después de la Madraza de Málaga (1334), de carácter privado (3) de la que no existen restos en la actualidad.

Situada en una localización privilegiada de la ciudad, junto a la Mezquita Mayor y la Alcaicería (4), la zona más noble del comercio en su época, hoy en día su ubicación se enclava en pleno centro histórico de Granada, en la calle Oficios, junto a la Capilla Real y la Catedral, en el área peatonal situada entre la Gran Vía, las calles Reyes Católicos y Zacatín y la plaza de Bibarrambla, lo que la sitúa en un área destacada para la vida cultural y turística de la ciudad (Figura 1).

Se trata de un inmueble histórico con protección de Bien de Interés Cultural (BIC), declarado Monumento histórico-artístico en 1922 (6-VII-1922) (6).

A diferencia de otros casos, la ocupación del edificio ha sido continua, estando dedicado a diversos usos, además del universitario, ocupado hasta 1499, destaca el acogido en el año 1500, como Casa del Cabildo (Ayuntamiento) (7) tras la conquista de la ciudad, cuando fue cedido por el rey Fernando II de Aragón. Desde entonces fue transformándose por completo hasta alcanzar su actual fisonomía barroca «en la década 1720-1730, que supuso la casi total desaparición de la Madraza Nazarí» (3), tras ser demolidos en gran parte los restos de la construcción árabe. En su interior el Salón de Caballeros XXIV, con su destacada armadura mudéjar de inicios del siglo xvI, es testimonio de ese momento de la historia del edificio. Como casa consistorial se mantuvo hasta 1858 , año en el que se realizó el traslado a su actual emplazamiento en la Plaza del Carmen. Posteriormente fue enajenado y pasó a ser de uso privado como almacén de tejidos, hasta que, en 1939, fue adquirido de nuevo por el Ayuntamiento (2). Tras diversos usos administrativos, en 1976 retoma su vinculación con la Universidad de Granada, actual propietaria, siendo hoy la sede de la oficina de Extensión Universitaria y Centro de Cultura Contemporánea, uso compartido con la Real Academia de Bellas Artes de Nuestra Señora de las Angustias, institución artística fundada el 18 de enero de 1777, podría decirse que se ha recuperado la función original para la que se construyó el edificio en época nazarí.

Como consecuencia directa de tal diversidad se han sucedido numerosas intervenciones y adaptaciones según las necesidades y criterios de cada momento que ofrecen un gran interés en el ámbito de la investigación científica.

El estudio que aquí se presenta se centra en una localización concreta del edificio, la Sala del Oratorio de la Madraza, estancia que destaca por ser la única localización de su pasado islámico que se conserva hoy en día junto con los restos arqueológicos integrados en su planta baja. Además es el único vestigio que permite valorar la relación del edificio actual con la antigua Madraza Yusufiyya, pues a pesar de tener en cuenta las múltiples transformaciones sufridas en el edificio a partir del siglo xvi, como sala baja y capilla del Cabildo y, sobre todo, a partir de 1725, transformada en antesala baja y oratorio, se han podido recuperar, estudiar y tratar las yeserías originales existentes bajo el recubrimiento de sus muros mediante revocos y yeserías de diseño churrigueresco, descubiertas gracias a la intervención del profesor Francisco Fernández González (3).

Diversos estudios han permitido aclarar numerosas incógnitas, ocultas bajo las sucesivas capas y alteraciones formales sufridas, que ponen de manifiesto el interés por investigar un edificio que fue emblema en su época y también lo es en la actualidad.

Tal es así que el mencionado interés se extiende y abarca desde sus orígenes históricos como primera universidad de la ciudad (3), (8), (9), (10), (11), hasta la información que

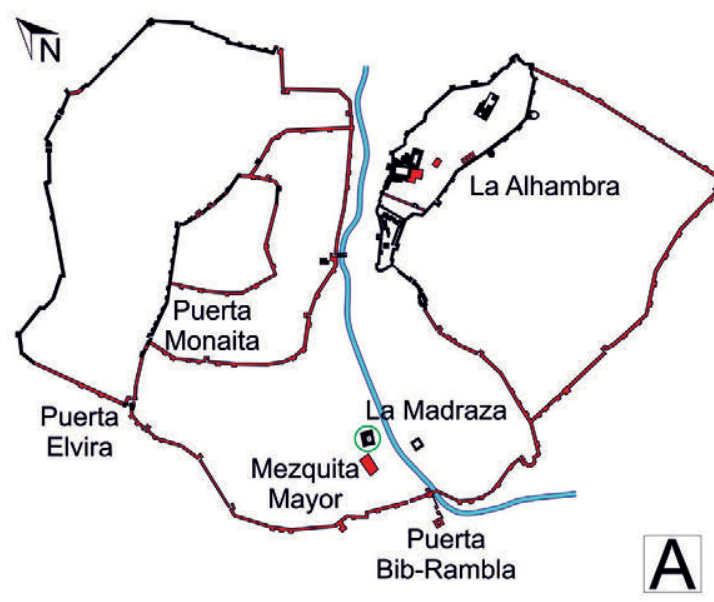

Construcciones existentes

Construcciones desaparecidas
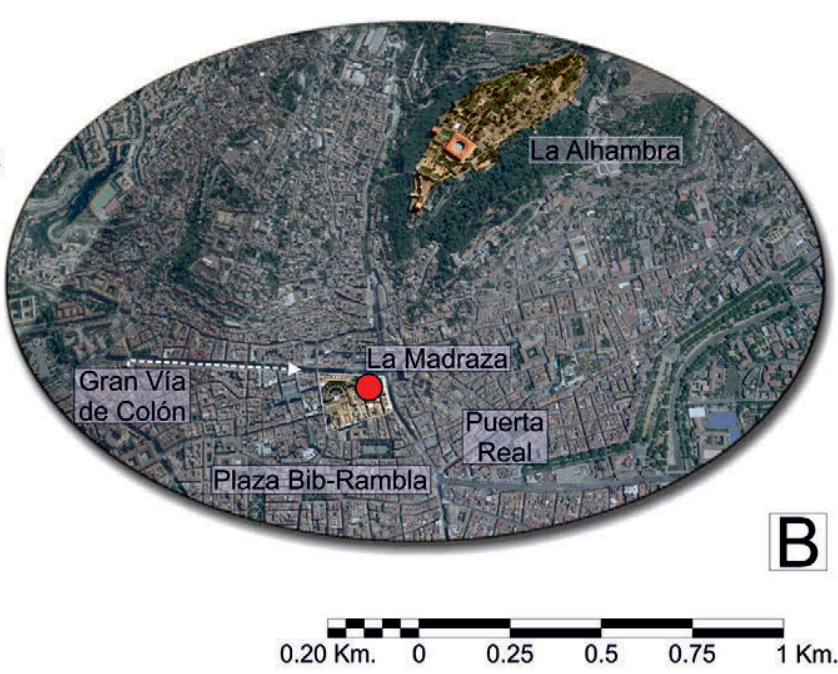

Figura 1. Localización del edificio en plano de la época árabe, adaptado de (5) y su contexto actual. 
ofrecen las epigrafías que contienen en la actualidad gran parte de los paramentos del oratorio y en su momento también su fachada y que han sido objeto de estudio por parte de (12), (13), (14), (15), (16) sin olvidar la trascendencia que ofrece el contexto urbano y de territorio (17), (18), (19), así como su primera configuración constructiva y posterior evolución, para los que se han desarrollado trabajos de arqueología por (20), (21), (22), (23), (24), permitiendo conocer la implicación con la arquitectura de distintas épocas, hasta más recientemente un levantamiento planimétrico actual de (25) y los estudios relacionados con las intervenciones en el campo de la restauración y rehabilitación del edificio (26), (27) con objeto de adecuarlo a las exigencias normativas y de uso actuales, que se han llevado a cabo en investigaciones muy exclusivas y singulares en el desarrollo de los estudios previos y de caracterización de sus materiales incluso estableciendo protocolos de actuación (28), destacando los realizados sobre las yeserías y pinturas existentes (29), (30), (31), (32), (33).

En concreto sobre los materiales, las técnicas utilizadas en su caracterización han permitido determinar la originalidad o no de algunos de los elementos visibles en la actualidad (30), (34), (35) y a su vez ha posibilitado la aplicación de las técnicas adecuadas para su recuperación.

El estudio está basado en la localización de alteraciones y su posicionamiento gráfico, estudio de materiales de alteración y medición de parámetros físicos (humedad y temperatura) para la mejor determinación de las propuestas restauradoras.

\section{MATERIALES Y MÉTODOS}

\subsection{Materiales}

Aunque no se dispone de excesivos datos sobre el edificio original tal y como se construyó, sí se ha podido confirmar complementando las fuentes escritas con los recientes estudios arqueológicos, cuál era la estructura inicial (8), (16), (20), (21), (22), (23). Según (36), la madraza granadina evidencia en su composición formal la influencia de la arquitectura norteafricana, disponiendo los espacios que lo componen en torno a un patio de moderadas dimensiones, rodeado por una galería con vestíbulo de entrada y el oratorio. En planta baja, según deducen las excavaciones realizadas (16), (23), (37), existían además varias salas para la docencia, las letrinas y la escalera de acceso al nivel superior. La planta alta, en su etapa inicial, daba acceso a través de un estrecho corredor en torno a la galería, a las celdas de los estudiantes. En este mismo nivel se encontraba también la biblioteca, relacionada con la institución, ofreciendo de esta forma un programa completo para el uso desarrollado (Figura 2).

Centrándonos en el Oratorio, éste se ubica en el lado opuesto de lo que fue en su momento el acceso al edificio, su entrada se realiza a través de dos finas columnas de mármol blanco sobre las que apoya un arco de herradura con alfiz y trasdós angrelado, decorado con atauriques e inscripciones iguales a las yeserías de su interior (38).

Siendo de planta cuadrada, la estructura octogonal del espacio visible se consigue por medio de cuatro pechinas (trompas), situadas en las esquinas del nivel inferior de los muros, decoradas con mocárabes. A partir de aquí todos sus frentes se articulan en diferentes niveles decorativos. En la parte baja de los muros se dispone de 6 arcos de herradura, enmarcados por alfiz y en el nivel superior un friso de dieciséis ventanas en arco con celosías que permiten el paso de la luz a su través disponiendo sobre ellas una cornisa de mocárabes (38).

En dimensiones, considerando que la estancia no ha sufrido modificaciones se reconocía una medida exterior de 6,84 metros de lado (14), con una superficie útil de 46,20 m² y construida de $61,50 \mathrm{~m}^{2}$ (16). En altura, coincidiendo con el edificio se reconocen 15 metros de altura máxima.

Originalmente el espacio se cubría con una techumbre de madera, con decoración de lazo, policromada y adornada con racimos de mocárabes. A mediados de siglo la cubierta del oratorio fue destruida por un incendio y en 1893 se construyó de nuevo. La intervención consistió en la cubrición con un alfarje de madera con abertura cenital, lo que permite la entrada de luz natural a través de la linterna de trazado octogonal, que en origen se conseguía a través del nivel de ventanas.

Frente a la entrada del oratorio se encuentra el mihrab, con menos profundidad en la actualidad, su acceso, al igual que la entrada, se realiza a través de un arco de herradura ondulado. En su interior no quedan restos de decoración.

En relación con su composición constructiva y material, en el Oratorio las investigaciones llevadas a cabo por (26), (28), (31) establecen una estructura compuesta por fábricas mixtas de ladrillo y mampostería. En concreto se ha determinado en algunas zonas, tras el picado del revestimiento de mortero de cemento, la distinta composición material de los muros, destacando la fábrica de ladrillo, la mampostería e incluso el tapial, todo ello debido a las modificaciones y alteraciones que ha acogido el edificio durante siglos. En todos los casos se encuentran revestidos con yeserías policromadas.

Su estructura horizontal se compone básicamente de alfarjes de madera entre los que se han encontrado consolidaciones más recientes realizadas con otros sistemas estructurales de acero y hormigón.

La cubierta, según describe (26), se resolvía como amalgama de diversos materiales dispuestos sobre y en el perímetro que delimita la planta del Oratorio, reconociendo cubierta de teja árabe sobre tablero de rasilla y tabicones, cubierta acristalada con perfilería metálica, cubierta plana de pavés y en la linterna cubierta con escamas de pizarra sobre tablazón y armadura de pares, que provienen en su mayoría de las intervenciones realizadas en el siglo xIx. Además se comprueba en su configuración formal como los elementos adosados del propio edificio y las construcciones que lo delimitan parcialmente en las fachadas Sur y Oeste se disponen a mayor altura en todos los casos.

Estas cuestiones de interés serán destacadas en el análisis realizado sobre el estado de deterioro.

\subsection{Metodología}

El inicio de los estudios desarrollados en la presente investigación tiene su origen en los análisis previos e intervenciones realizadas tanto a nivel arqueológico como en 

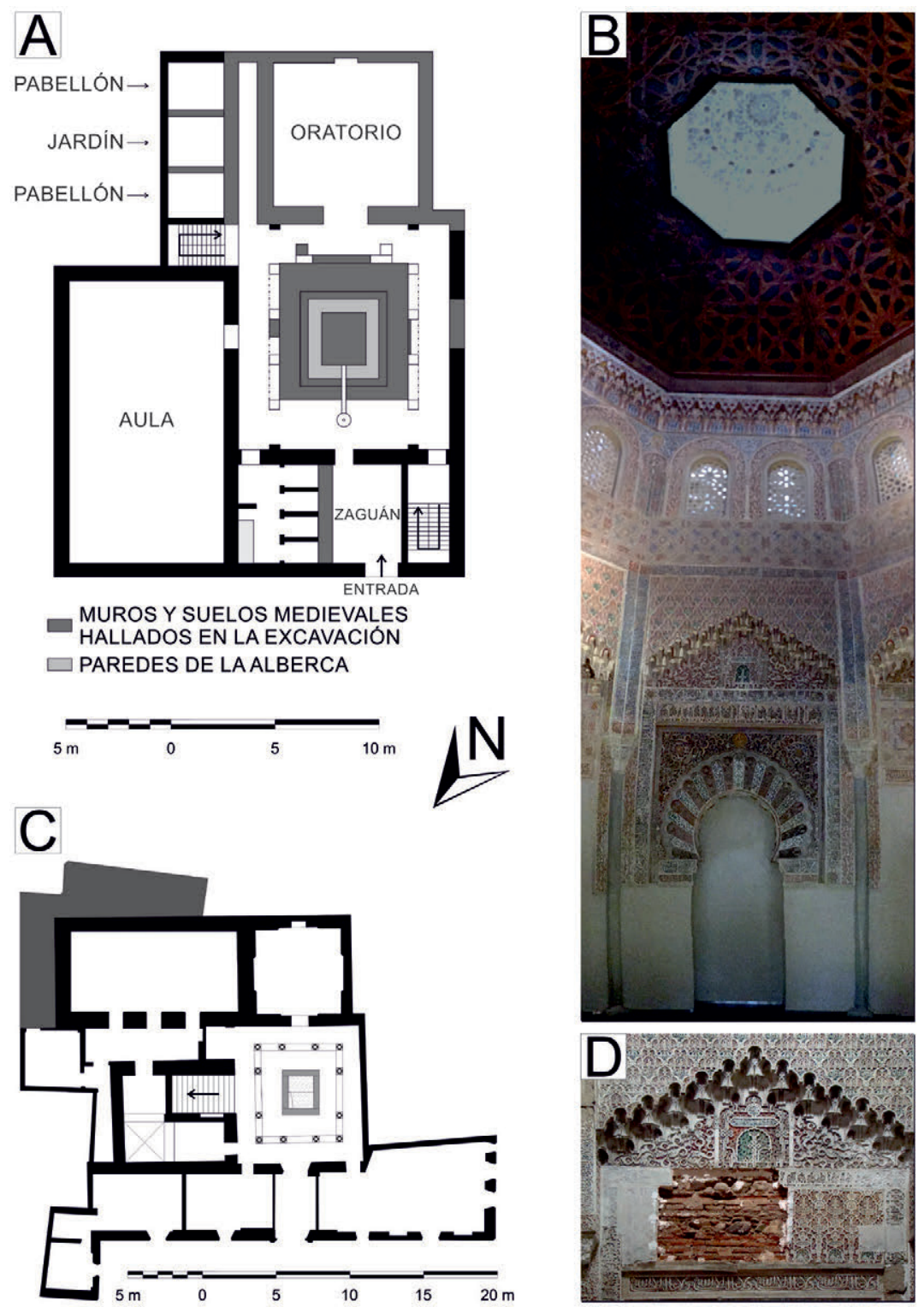

Figura 2. (a) Plano de planta del edificio original [adaptado de (16)]; (b) Fotografía (del oratorio, vista del Mihrab (foto de los autores); (c) Plano de planta del edificio actual [adaptado de (37)]; (d) Detalle de estado composicional de muro original. [Fuente propia].

actuaciones rehabilitadoras y restauradoras, en las cuales se llevan a cabo trabajos de detalle de distintas localizaciones y elementos en el edificio, según se ha comentado en el apartado 2.1. Materiales.

El reconocimiento del estado de conservación en esta singular localización se complementa con los estudios realizados en las yeserías (29), (30), con los cuales se comparten algunas fases de la metodología seguida. En concreto este trabajo se ha basado en una serie de protocolos y/o fases con una clara direccionalidad en la determinación de las intervenciones restauradoras a partir del alto nivel de protección patrimonial del edificio, siendo los siguientes: inspección visual del área de intervención (a), documentación gráfica y fotográfica de la totalidad del oratorio (b), clasificación-subdivisión del oratorio en áreas (sectores y niveles), lo que supone un protocolo espacial de intervención (c), reproducción gráfica pormenorizada de las áreas (d), posicionamiento x-y de los deterioros, atendiendo la definición y el léxico de los mismos según Documento NOR-
MAL 1/88 (e) (39), y cuantificación numérica de los deterioros (f). A partir de aquí se pueden extraer las muestras necesarias para realizar el análisis científico de las mismas (g), y finalmente propuesta de intervención restauradora $(\mathrm{h})$.

En concreto ésta consistió en un primer momento en una inspección visual meticulosa y prolongada, en la que se tomaron datos in situ de caracterización y dimensionado preliminar, así como los tipos y grados de alteración, su localización y su orientación en la estancia. Utilizando para ello lupa binocular, escalímetro, distanciómetro láser, etc. A continuación se documentó fotográficamente la estancia en toda su altura y desarrollo, procurando mantener la ortogonalidad, generando gran cantidad de material gráfico. Para ello se utilizó una cámara marca CANON EOS 5DS R, con sensor CMOS $36 \times$ $24 \mathrm{~mm}$ con 50,6 megapíxeles de resolución y relación de aspecto $3: 2$, rango de funcionamiento $\mathrm{AF}-2-18 \mathrm{EV}$ (a $20^{\circ} \mathrm{C}$ y 100 ISO), con objetivo profesional 24-105 mm. La cámara fue nivelada sobre el trípode en cada sesión fotográfica. 
Es importante destacar que tratándose de una superficie totalmente decorada y en la que existen numerosos elementos singulares (Figura 2 (c), (por ejemplo, lacerías, arabescos, mocárabes, etc.) nos encontramos con una dificultad añadida, al tener que trabajar en zonas con una elevada semejanza gráfica al tratarse de elementos decorativos que se repetían sistemáticamente (Figura 2 (c) y Figura 3) lo que hizo necesario desarrollar otras fases durante la toma de datos que permitieron sistematizar el estudio de toda la estancia.

Para ello se subdividió la estancia del oratorio en distintas áreas, establecidas en niveles y sectores, coincidiendo con diversas discontinuidades de tipo formal, ornamental o constructivo.

En horizontal y siguiendo el trazado de la planta octogonal, se dispusieron 8 sectores numerados de forma consecutiva del 1 al 8 (el 1 corresponde con el frente de entrada al oratorio y sigue la numeración de izquierda a derecha) a los que se añadieron zonas complementarias dependiendo del nivel en que se realizara la inspección (esquinas y mocárabes). En sección vertical, se subdividió la altura de la estancia en 6 niveles denominados de arriba abajo con las letras A, B, C, D, E y F, haciendo coincidir cada uno de ellos con las discontinuida- des formales presentes. En ese mismo orden se identifican los siguientes niveles decorativos: mocárabes (A), ventanas (B), cenefa (C), faldón (D), y en la parte baja los niveles E y F.

En la Figura 3 se muestra gráficamente la descomposición de las zonas de estudio.

En una siguiente fase se realizaron reproducciones gráficas de los distintos paramentos, en los que se marcaron los diferentes deterioros observados en el estado real de las yeserías, tanto desde un punto de vista conservativo (fisuras, sales, deformaciones, etc.) como gráfico (mocárabes, lacerías, epigrafía, etc.) que de otra forma podrían pasar totalmente inadvertidos.

Para finalmente realizar los dibujos de los diferentes paramentos a los que incorporar con total exactitud la información conseguida sobre el estado de conservación, como se muestra en la Figura 4.

Con objeto de facilitar la comprensión de la toma de datos fue necesario realizar diversos esquemas para cada sector y nivel, evitando así la excesiva superposición de información, sobre todo, en las zonas más afectadas. Distinguiendo entre aquellos dete-

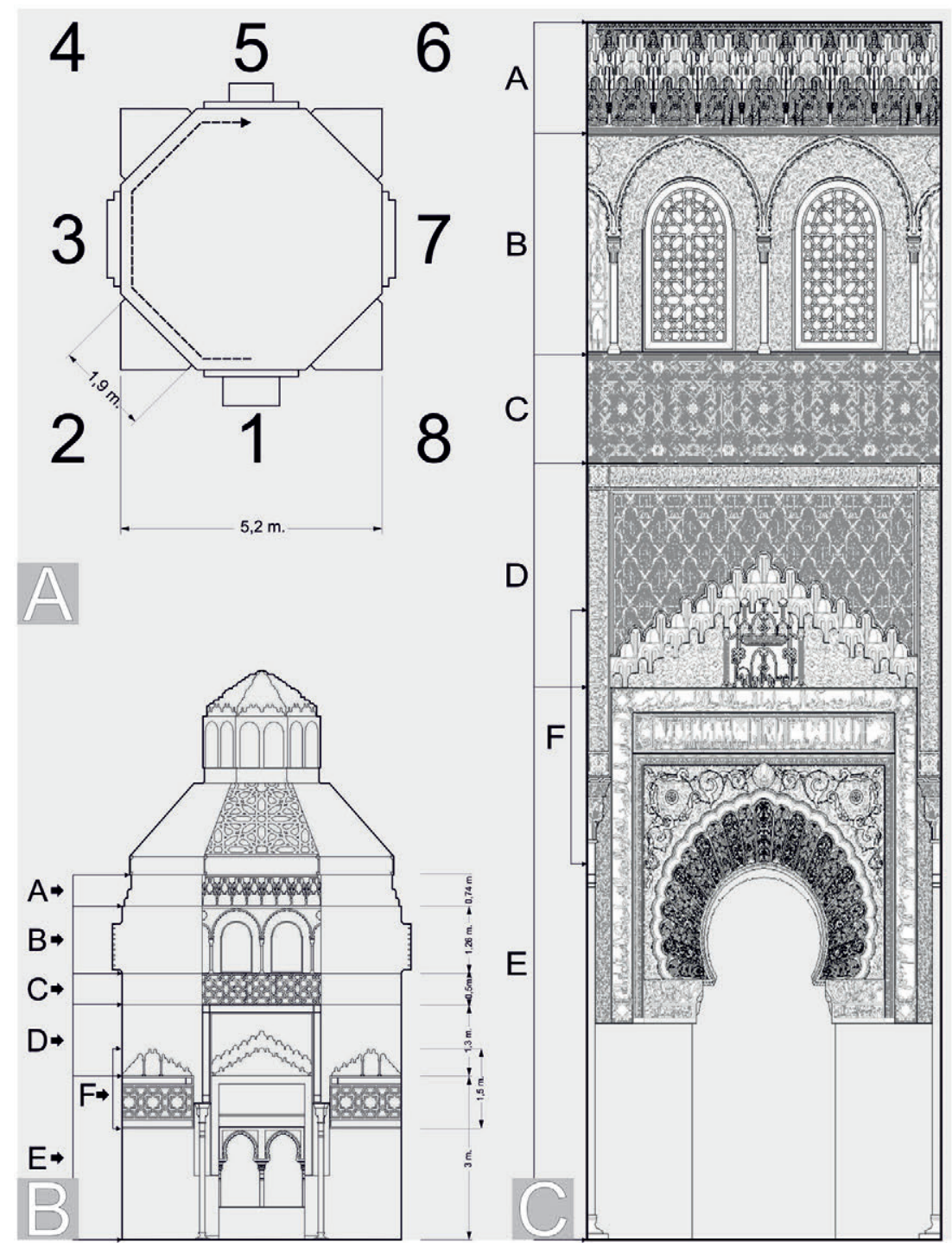

Figura 3. Áreas de subdivisión del oratorio para su estudio. (a) Sectores; (b y c) Niveles. [Fuente propia, basado en (37)]. 
rioros que suponían pérdida de cohesión y material (1) y aquellos que no suponían ninguna pérdida de cohesión ni material (2).

Una vez compilada toda la información para cada zona se reveló el estado real/actual, los tipos y grados de alteración, su ubicación vertical y horizontal, así como su orientación en el oratorio. Se estableció una tabla de deterioros para cada sector y nivel con una escala del 1 al 5 , con la que poder determinar el estado real ( 1 = menor afección; 5 = máxima afección; 0 = no afección).

Por último, en la estancia se realizaron controles de humedad absoluta en superficie en las yeserías y control de temperatura y humedad relativa, de igual forma tras la toma de muestras en algunos casos, se realizaron análisis de DRX y microscopía electrónica.

En el primer caso se utilizó un medidor de humedad absoluta de materiales en superficie, marca PCE Instruments, modelo PCE-PMI2, provisto de un sensor capacitativo. Por otra parte, los valores ambientales de temperatura y humedad relativa se realizaron con un termo higrómetro ambiental marca PCE Instruments, modelo PCE-310 basado en un sensor de resistencia eléctrica.

Las muestras de difracción de rayos $\mathrm{X}$ se analizaron utilizando un difractómetro Bruker D8 Avance con la geometría 2-theta, un detector de radiación de cobre y LYNXEYE, perteneciente al Departamento de Mineralogía de la Universidad de Granada. El análisis de la microestructura se realizó con un microscopio electrónico de barrido de la CIC, modelo Hitachi S-510, con un voltaje de aceleración de $25 \mathrm{kV}$, ampliación 20 a $150.000,5 \mathrm{~mm}$ de distancia de trabajo, detector EDX Röntec M Series, Edwin, $\mathrm{Si}$ (Li), y el sistema de microanálisis Edwin Röntec, perteneciente al Centro de Instrumentación Científica (CIC) de la Universidad de Granada.

\section{RESULTADOS. ESTADO DE CONSERVACIÓN. TIPOLOGÍA, ANÁLISIS Y EVALUACIÓN}

\subsection{Tipología de deterioros}

En este apartado se exponen los resultados obtenidos tras la aplicación de la metodología expuesta y el reconocimiento y análisis desarrollado.

En concreto, a continuación, en la Figura 5 se compone una muestra de los deterioros reconocidos en el Oratorio, clasificados en Tipo 1 y Tipo 2.

\subsubsection{Deterioros Tipo 1}

Este tipo de deterioro, ilustrado en la Figura 5 (a, b, c, d, e, f) incluye fenómenos tales como fracturas (fisuras), falta de soporte y oquedades, en definitiva todas las formas de pérdida de material observadas (fisuras, oquedades, pérdida de soporte...), algunos derivados de la naturaleza del propio material.

\section{Eflorescencias}

La extensión y abundancia de las eflorescencias detectadas es variable, así como su localización, destacando su posicionamiento en los niveles más elevados del oratorio (A-B, sectores 1 a 8), ello es consecuencia de los problemas de evacuación de agua que presenta la cubierta antes de su reparación, derivando agua de escorrentía en los niveles inferiores que producen «canales» y zonas afiladas en el soporte. Un gran porcentaje se localiza en el nivel B (sectores 5, 6 y 7) próximo a las áreas de filtración de humedades y percolación de agua, acelerando la disolución de los soportes y posterior precipitación en forma de sales, en menor medida en el nivel $\mathrm{C}$ (sectores 3 y 6) y, finalmente, las eflorescencias aparecen

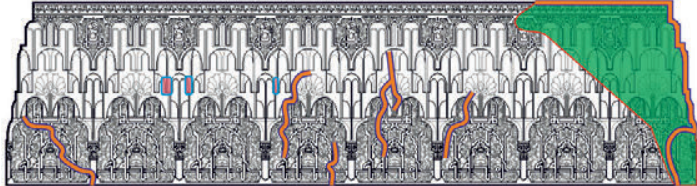

NIVEL A. SECTOR 1

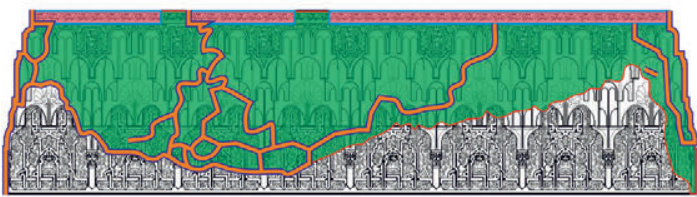

NIVEL A. SECTOR 2

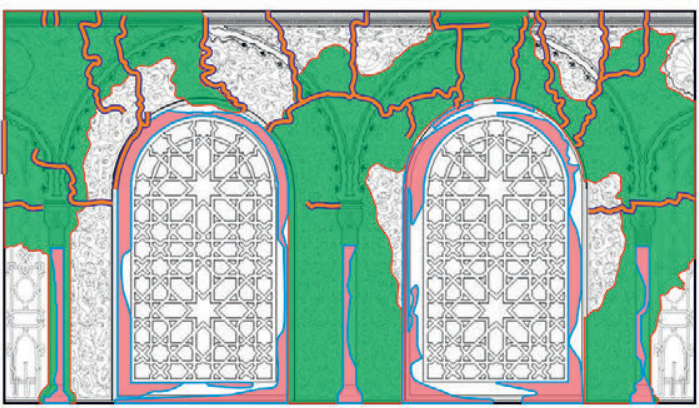

TOQUEDADES

$\square$ FALTA DE SOPORTE

= FISURAS Y GRIETAS

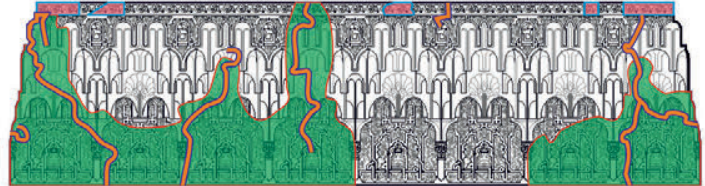

NIVEL A. SECTOR 3

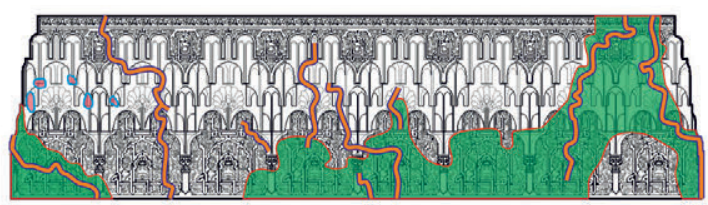

NIVEL A. SECTOR 4

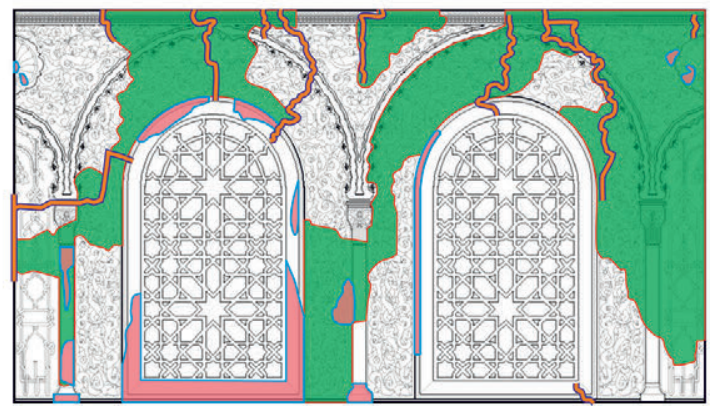

NIVEL B. SECTOR 4

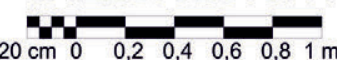

Figura 4. Ejemplo del levantamiento gráfico realizado en diferentes niveles y sectores con especificación de los deterioros correspondientes. 

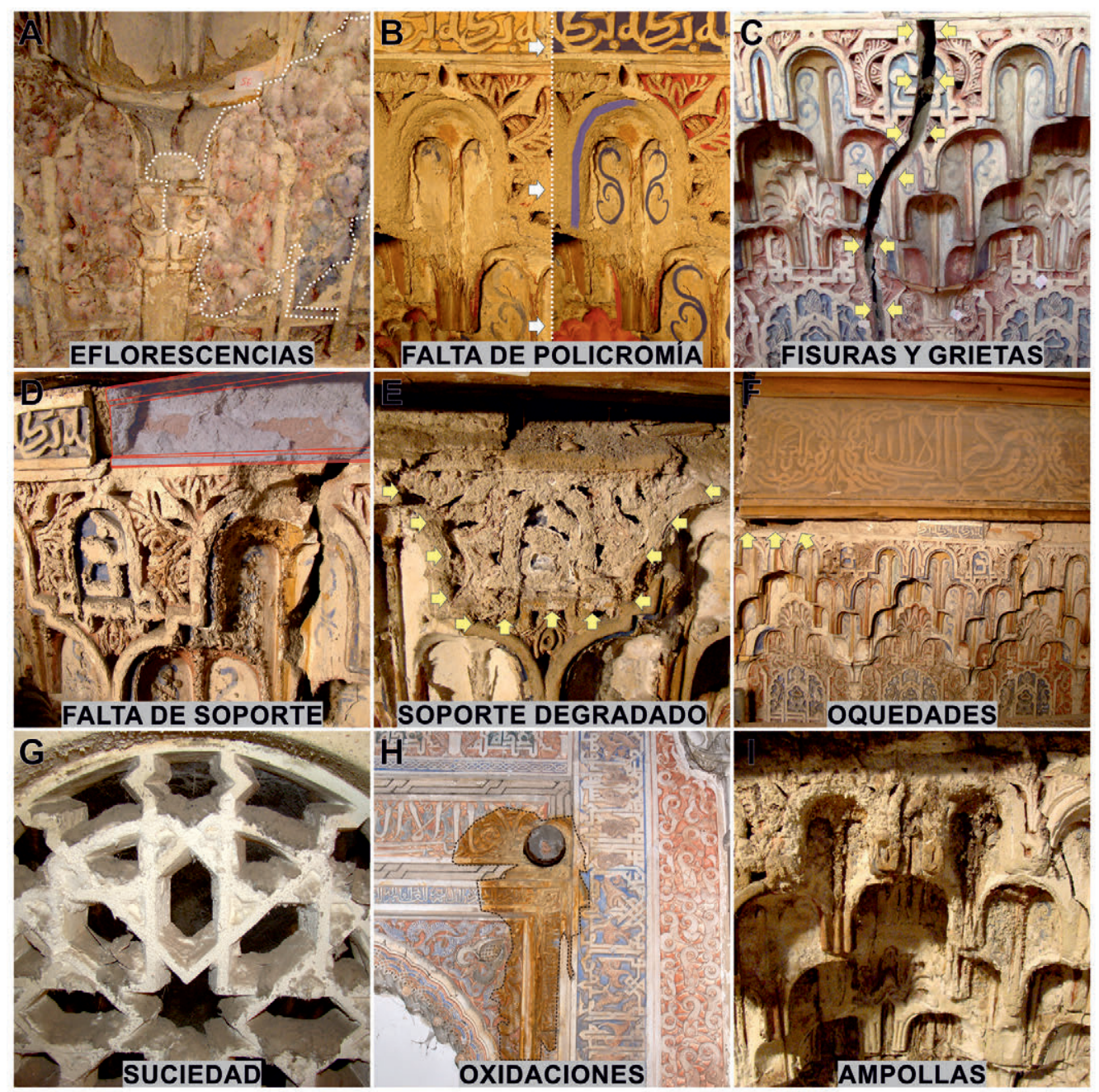

Figura 5. Deterioros Tipo 1 (a, b, c, d, e y f) y Deterioros Tipo 2 (g, h, i). [Fuente propia].

intermitentemente por el resto de los soportes decorados de la estancia, asociadas a fenómenos de capilaridad.

Junto con la presencia de manchas blanquecinas en la superficie, característica de las eflorescencias, se detectan áreas disgregadas y descohesionadas en los soportes, con extensión y profundidades considerables por la presencia de subeflorescencias. Tanto la composición del soporte (yeso y otros aditivos) como algunos materiales de reparación empleados (yesos, morteros bastardos y cemento tipo portland) son los causantes de la aparición de sales de hemidrita, epsomita y carbonato cálcico (Figura 6).

\section{Falta de policromía}

Se observan en todo el perímetro del oratorio alteraciones relacionadas con la policromía, visibles en diferentes grados y obviando los «repintes históricos» dado que forman parte del estrato pictórico que es objeto de estudio. Estos repintes incluso han permutado el tono original de numerosas áreas policromadas (29).

En general hay diferentes formas de alteración en los acabados polícromos de los yesos, todo ello sin obviar que las alteraciones se manifiestan en extensión y profundidad de

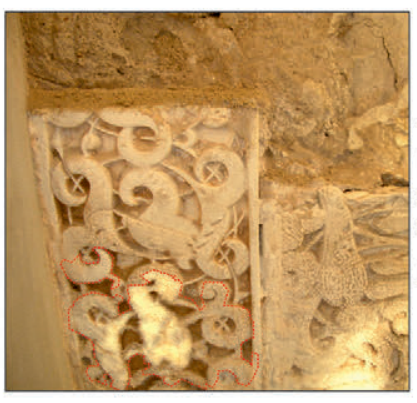

A
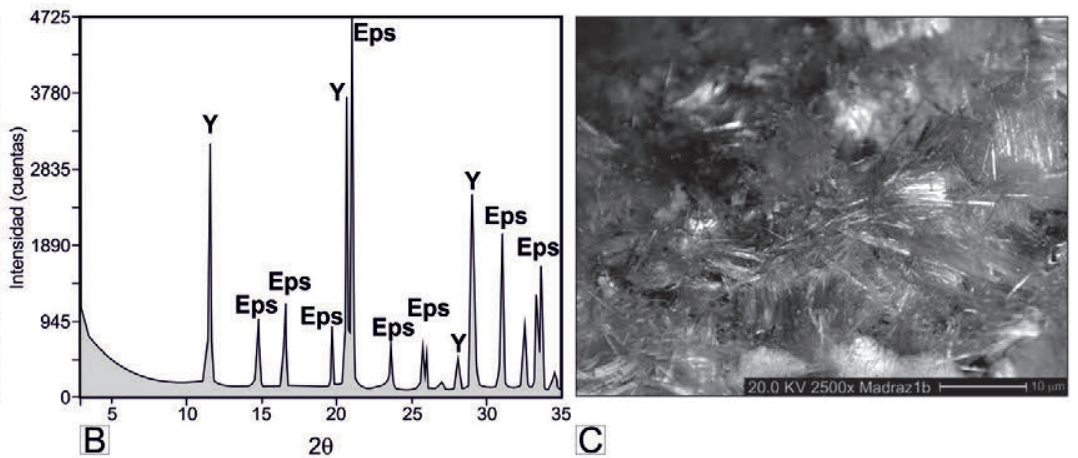

Figura 6. (a) Detalle de eflorescencias; (b) DRX de las eflorescencias, donde destaca la epsomita mayoritaria (Eps) junto al yeso (Y); (c) Macrofotografía SEM de la epsomita de las yeserías. [Fuente propia]. 
manera heterogénea. Por ejemplo, en la capa de preparación, enjalbegado o capa fina (generalmente con imprimación aislante) apta para recibir la policromía, son evidentes las faltas y lagunas, al igual que se detecta una falta de adhesividad de esta capa final con el soporte de yeso subyacente.

Lo habitual en la capa de policromía es que se entremezclen formas de alteración, destacando los lavados de la misma a consecuencia de agua de escorrentía desde la parte superior, precipitaciones de manchas de pigmentos y soporte de yeso en niveles inferiores, así como una importante presencia de eflorescencias y cristalizaciones de sales solubles en superficie con la consiguiente aparición de «velos» blanquecinos y desagregaciones. Por otra parte se puede inferir que la generalizada falta de cohesión de la película pictórica y subsecuentes desprendimientos en importantes áreas esté condicionada por las inestables condiciones ambientales de toda la sala, como queda de manifiesto en las mediciones de humedad absoluta y temperatura y humedad relativa en ambiente, cuyos datos más críticos corresponden a la medición del mes de marzo (marzo: $17,4 \pm 1,3{ }^{\circ} \mathrm{C}, 74,5 \pm 2,4 \% \mathrm{H}$ ).

En este caso, tratándose de una investigación sobre el estado de conservación se ha considerado la falta de policromías en toda la sala sin descartar aquellas zonas en las que siendo evidente la no originalidad (considerada para las áreas intervenidas en fechas posteriores a su construcción), son motivo de interés al estar incluidas en la zona de estudio y siendo útiles para la determinación de causas de deterioro.

Los sectores más afectados son el 3 y el 6, siendo el nivel E-F el que mayor deterioro presenta para este caso.

\section{Fisuras}

La presencia de fisuras en la mayoría de los sectores y niveles evidencia los esfuerzos no soportados que acogen estos elementos. Se reconocen de trazado variable y disperso, más frecuentes las verticales y en menor presencia horizontales e inclinadas. Coincidentes en su mayoría con el trazado gráfico y geométrico, juntas de unión, discontinuidades o menor espesor de placa. Aunque en ocasiones su trazo es aleatorio.

Se han considerado fisuras a todas las que únicamente rompen el acabado, independientemente de la abertura que tengan y grietas las que rompen tanto el acabado como el soporte.

La fisonomía de la fisuración es diferente según zonas. Así, por ejemplo, en los niveles de mocárabes (nivel A) la mayoría de las fisuras son verticales y subverticales, que en ocasiones convergen quedando unidas a través de fisuras horizontales o inclinadas.

En las cenefas (nivel C) la coincidencia del trazo con los motivos geométricos es mayoritaria. En el nivel de ventanas (nivel B) se observa mayor afección en los arcos que circundan los huecos de celosía y sus dinteles, marcando las zonas de descarga de dichos arcos.

Los faldones (nivel D) son el nivel menos afectado por este tipo de deterioro, siendo localizado en las juntas verticales que delimitan las jambas del resto de decoración del paño y de forma excepcional en algunos elementos decorativos.

Se destaca por su mayor gravedad el pórtico lateral en el sector 7, nivel E-F, en el que se observan fisuras de todas las tipo- logías comentadas, abertura de todas las juntas verticales de unión entre placas, partición de arcos de entrada, etc.

Las causas que las originan han sido preferentemente las presiones/esfuerzos ocasionados por cambios de temperatura de los materiales constitutivos y por tensiones derivadas del desplazamiento de la armadura de madera.

\section{Pérdidas de soporte, falta de adherencia al soporte y oquedades}

Son reseñables tanto las pérdidas de soporte en las yeserías como la falta de adhesión entre las capas que constituyen los paramentos decorados de yeso del oratorio. En ambos casos los fenómenos de disolución de los soportes y la aparición de sales precipitadas y subeflorescencias son las causas de alteración probables. De esta forma se detectan pérdidas de soporte en la línea epigráfica de los mocárabes bajo arrocabe, en el recercado de las celosías, en las pilastras y en los mocárabes esquineros.

Es significativa la pérdida de adhesión entre los diferentes estratos y el soporte de obra al observarse una separación de éstos en diversas áreas de la estancia, provocando oquedades, deformaciones y grandes «abombados» en los niveles externos y, en su caso, pérdida de soporte (mocárabes superiores de los sectores 2 y 6 en los niveles A y B).

\section{Soporte disgregado}

Se detectan diversas áreas con disgregación de soporte sin pérdida del mismo, motivadas por la falta de adecuadas condiciones termohigrométricas en el oratorio, subsecuente condensación de humedad ambiental y disolución progresiva de los soportes siendo evidentes en los niveles A y B principalmente. Las diferentes mediciones de superficie en zonas pulverulentas y disgregadas arrojaron valores muy elevados de humedad contenida en los materiales (yeserías).

Por una parte, las mediciones de humedad absoluta y temperatura y vapor de agua en el oratorio que fueron obtenidas sobre un muestreo de 30 puntos en superficie, cuyo valor medio $(\overline{\mathrm{X}})$ fue de 77,6 con desviación estándar $(\sigma)$ de 3,4, equivalen en términos generales a soportes húmedos y/o mojados, cuyas causas pueden estar determinadas por fenómenos de percolación de agua desde las cubiertas y cierres del oratorio (con mayor probabilidad), o por condensación de vapor de agua del ambiente. Por otro lado las medidas de temperatura y humedad relativa realizadas en el oratorio, en tres meses, fueron las siguientes: diciembre, $14 \pm 0,7^{\circ} \mathrm{C}, 71,4 \pm 2,4 \% \mathrm{H}$; marzo, $17,4 \pm 1,3{ }^{\circ} \mathrm{C}, 74,5 \pm 2,4 \% \mathrm{H}$; julio, $22 \pm 1,3{ }^{\circ} \mathrm{C}, 69,8 \pm 2,4 \% \mathrm{H}$.

Los datos de humedad en los soportes medidos ponen de manifiesto los procesos de disolución progresiva de los mismos y su disgregación, paralelamente, y a partir de las condiciones termohigrométricas ambientales medidas se pueden inferir caídas de temperatura, especialmente en la medición de marzo $(\approx 20 \%$ ), que hayan provocado condensación de vapor de agua en la superficie de los materiales, aumentando la presencia, en éstos, de agua líquida.

\subsubsection{Deterioros Tipo 2}

En este grupo se han incluido aquellos deterioros que no producen pérdida de material pero que pueden acelerar otras alteraciones Figura 5 (g, h, i). 
Pueden reseñarse los grandes acúmulos de polvo superficial y otros materiales que generan suciedad (por ejemplo, excrementos de insectos y de murciélagos). Las partes menos accesibles del oratorio son las más dañadas y las que muestran un mayor ennegrecimiento de los soportes.

Algunas intervenciones de restauración incorrectas destacan por su tosquedad y falta de pericia. Tal es el caso de las reintegraciones de soportes realizadas en algunos mocárabes esquineros o paños de yeserías, las cuales incorporan masillas de yeso que ocultan parte de los yesos tallados originales, además de no reproducir determinadas líneas maestras del diseño de los mismos. Se han observado firmas y grafiti de intervenciones restauradoras anteriores que alteran en cierta medida el soporte original.

Se constatan zonas de ampollas (Figura 5 i), levantamientos y ennegrecimientos de las policromías, soportes de yeso y enjalbegados en las zonas altas de las yeserías provocadas por el incendio que arrasó la armadura original durante el siglo XIX (28).

De igual manera se menciona la presencia de algunos elementos metálicos oxidados en la estancia (por ejemplo, piezas metálicas para sujeción de relieves, clavos y puntas de hierro que sujetaban cables y otras instalaciones, etc.), los cuales producen manchas de fuerte coloración pardo-rojiza al soporte decorado de yeso (Figura $5 \mathrm{~h}$ ).

Para este grupo la localización es totalmente aleatoria y anecdótica, por lo que no se realizó para esta investigación el mapeo correspondiente al no poder constatar la correlación de deterioros con total fiabilidad.

\subsection{Análisis y evaluación de las tipologías de deterioro}

Los datos obtenidos por la meticulosa y prolongada inspección visual han permitido desvelar el estado de conservación del oratorio, así como los tipos y grados de alteración, su ubicación en los distintos niveles y sectores y su correlación con el entorno constructivo y de materiales existentes.

En la Figura 7 se recogen para los deterioros del grupo 1, el grado de afección resultante, clasificados por tipo de deterioro y nivel, así como su representación gráfica para una mejor interpretación.

Los deterioros más representativos ( $>20 \%$ ) de mayor a menor intensidad han sido las oquedades, fisuras y eflorescencias, que se reconocen en toda la estancia, aunque como se muestra en la Figura 7 perfectamente localizados.
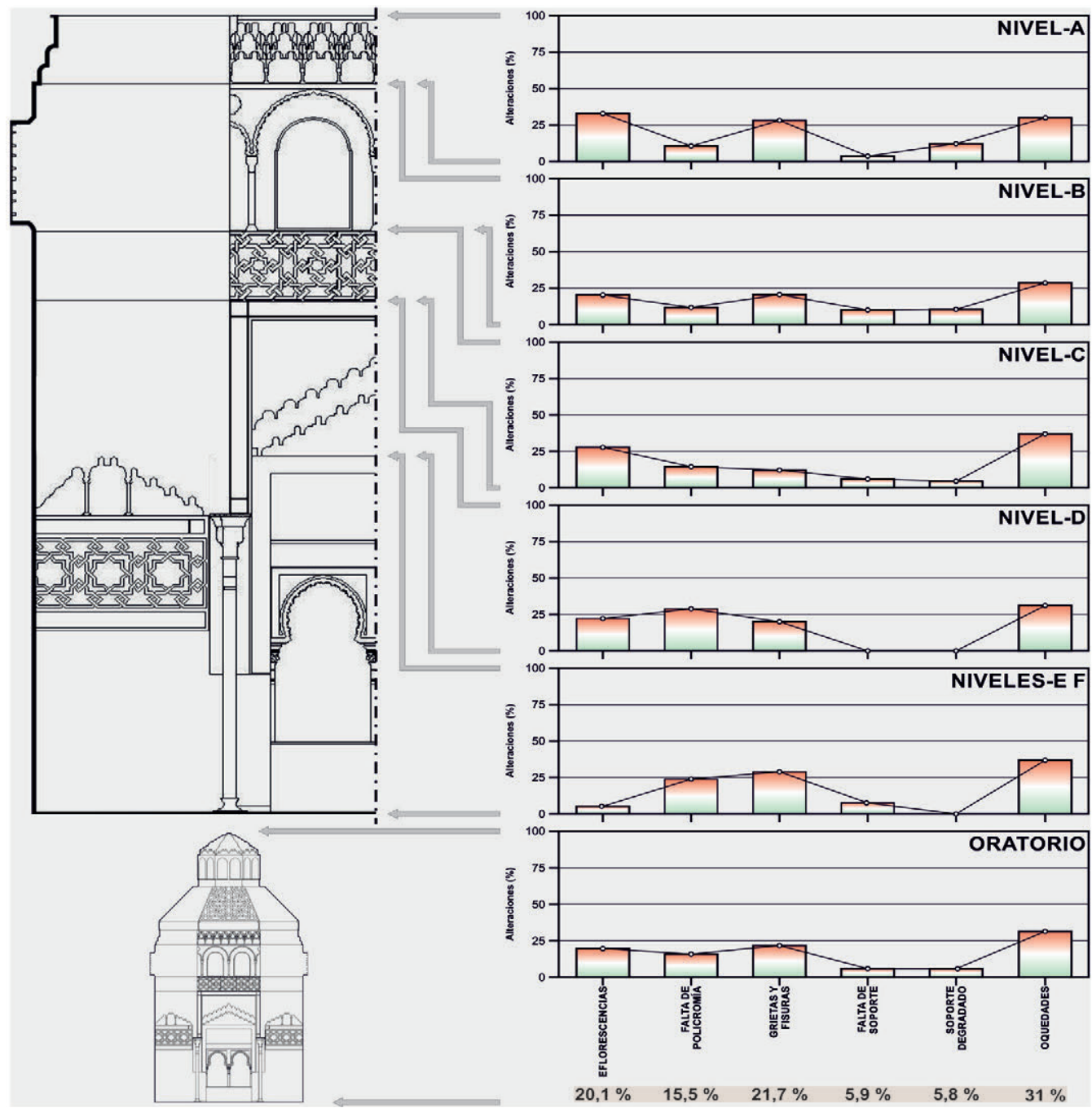

Figura 7. Representación gráfica del estado de deterioros Tipo 1 localizados en el oratorio y sus diferentes niveles (A, B, C, D, E-F). 
Por sectores el más afectado es el sector 6, seguido del 7, el 3 y el 2, ubicados de forma consecutiva dos a dos en la diagonal de la estancia. Por último, para los niveles se destaca tras su evaluación la máxima alteración en los niveles A y B, respectivamente siendo señalados también los niveles $\mathrm{C}$ y E-F como afectados en porcentaje elevado.

Conocida la relación que las zonas más afectadas tienen con el resto del edificio, se constata la implicación que para las zonas altas tiene la amalgama de soluciones constructivas de la cubierta (situadas a cota superior) tanto de las estancias colindantes como de los edificios medianeros que delimitan el oratorio y las filtraciones por percolación que han favorecido de forma indiscriminada la filtración de agua y como consecuencia las alteraciones conocidas.

\section{CONCLUSIONES}

Los resultados obtenidos permiten establecer como causa más probable de deterioros mecánicos la ubicación de elementos adosados a la construcción y más concretamente la solución adoptada en los mismos, reconociéndose puntos singulares (encuentros en todo el perímetro, distintos niveles de evacuación de aguas...), así como causas relacionadas con su configuración estructural y constructiva (muros de carga, forjados...). Por otra parte, los valores de humedad absoluta de las yeserías y las condiciones termohigrométricas de la estancia ponen de manifiesto la presencia de causas de disgregación en los soportes, aceleración de los procesos de fisuración, pérdidas de policromías y aparición de eflorescencias.

Cuantitativamente las principales alteraciones detectadas son las oquedades, grietas y fisuras y eflorescencias, presentes en todo el oratorio. En general se puede precisar una mayor abundancia en los niveles A y B, así como en C, E-F. Incidiendo en el origen de las alteraciones, éstas pueden estar motivadas por los esfuerzos mecánicos, humedades de percolación y condensación que se producen en las zonas altas del oratorio, y las humedades, probablemente por capilaridad y condensación del nivel más bajo.

Por otra parte corresponde destacar que el proceso metodológico realizado, el cual se ha basado en fases secuenciales para la determinación de las intervenciones restauradoras, puede ser aplicado en la intervención conservadora de otros edificios con altos niveles de protección patrimonial, lo que permite determinar con precisión las intervenciones restauradoras y conservadoras de los mismos.

\section{AGRADECIMIENTOS}

La investigación presentada en este documento se ha desarrollado en el marco del PROYECTO REMINE MSCA, Horizon 2020 (RISE) y los Grupos de Investigación RNM 0179 y HUM 629 de la Junta de Andalucía.

Nuestro agradecimiento al Dr. Arquitecto D. Pedro Salmerón Escobar, por la ayuda prestada facilitando la base de planimetría utilizada, y que pertenece al proyecto «Recuperación del Palacio de la Madraza como espacio cultural de la Universidad de Granada».

Igualmente, nuestro agradecimiento al Instituto de Conservación y Restauración de Bienes Culturales de la Fundación CICOP.

Los autores agradecen a los revisores las sugerencias propuestas a este trabajo de investigación.

\section{REFERENCIAS}

(1) Encyclopædia Iranica (2011). Education iv. The medieval madrasa. http://www.iranicaonline.org/articles/education-ivthe-medieval-madrasa.

(2) Universidad de Granada. Centro de Cultura Contemporánea. http://veu.ugr.es/pages/cecuco.

(3) Cruz Cabrera, J. P., Gómez-Moreno Calera, J. M. (2007). Estudio histórico-artístico del palacio de la Madraza, antigua casa del cabildo de Granada. En López Guzmán, R., Díez Jorge, M. ${ }^{a}$ E. (Eds.), La Madraza: pasado, presente y futuro (pp. 43-137). Granada: Editorial Universidad de Granada.

(4) Seco de Lucena Paredes, L. (1975). La Granada nazarí del siglo xv. Granada: Ed. Patronato de La Alhambra.

(5) Seco de Lucena, L. (1910). Plano de Granada Árabe. http://image.slidesharecdn.com/unavisitaagranada-elalbayznpuertasymurallas-091016180956-phpappo1/95/una-visita-a-granada-el-albayzn-puertas-y-murallas-2-728.jpg?cb=1255716765.

(6) De Parrondo Acero, C., et al. (1973). Inventario del Patrimonio Artístico y Arqueológico de España. Declaraciones de Monumentos y Conjuntos Histórico-Artísticos, Parajes Pintorescos y Jardines Artísticos. http://es.calameo.com/ books/oooo7533542eae8of98f5.

(7) Moreno Garzón, L. (1984). Carta Real de Merced a la Ciudad de Granada determinando la organización del Cabildo, Año de Mil Quinientos. Ed. Facsímil. Granada.

(8) Cabanelas Rodríguez, D. (1977). Inscripción poética de la antigua madraza granadina. Miscelánea de Estudios Árabes y Hebraicos, 26: 7-26.

(9) Cabanelas Rodríguez, D. (1989). La Madraza árabe de Granada y su suerte en época cristiana. Cuadernos de la Alhambra (24): 29-54.

(10) Cabanelas Rodríguez, D. (1990). La antigua Madraza granadina y su ulterior destino en época cristiana. Boletín de la Real Academia de Bellas Artes Nuestra Señora de las Angustias, 1: 27-49.

(11) Cabanelas Rodríguez, D. (1994). La Madraza árabe de Granada. En Universidad y ciudad: la Universidad en la historia y la cultura de Granada, 408 pp. Granada: Universidad de Granada.

(12) Acién Almansa, M. (1995). Inscripción de la portada de la Madraza. Catálogo de Exposición. En Arte islámico en Granada. Propuesta para un Museo de la Alhambra (pp. 337-339).

(13) Almagro Cárdenas, A. (1877). Inscripciones árabes de Granada y apuntes arqueológicos sobre la Madraza. Granada: Facsímiles Extramuros Edición. http://www.extramuros.es/mas_informacion_sobre_Estudio-sobre-las-inscripciones-arabes-de-Granada_isbn_978-84-9862-497-7__Eo9-0o886. 
(14) Almagro Cárdenas, A. (1898). El mihrab de la almadraza granadina, recientemente descubierto. Boletín de la Real Academia de la Historia, tomo 27, cuaderno III, julio-septiembre: 490-498.

(15) Almagro Basch, M. (1879). Estudio sobre las inscripciones árabes de Granada con un apéndice sobre su Madraza o Universidad árabe. Granada: Sabatel.

(16) Sarr Marroco, B., Mattei, L. (2009). La madraza Yusufiyya en época andalusí: un diálogo entre las fuentes árabes escritas y arqueológicas. Arqueología y Territorio Medieval, 16: 53-74.

(17) Seco de Lucena Paredes, L. (1956). El Hayyib Ridwan, la Madraza de Granada y las murallas del Albayzín. Al-Ándalus. Revista de las Escuelas de Estudios Árabes de Madrid y Granada, 21(1): 285-296.

(18) Malpica Cuello, A., García Porras, A. (2011). Las ciudades nazaríes. Granada: Universidad de Granada.

(19) Olmedo Sánchez, Y. (2002). Arquitectura y urbanismo en la Granada del Barroco Tardío (1667-1750). Córdoba: Universidad de Córdoba.

(20) Malpica Cuello, A., Martín Civantos, J. M., Mattei, L., González Escudero, Á., y Ruiz Jiménez, A. (2007). Intervención arqueológica de apoyo a la restauración del Palacio de la Madraza, Granada (2006-2007). En Anuario arqueológico de Andalucía. Sevilla: Consejería de Cultura, Junta de Andalucía.

(21) Malpica Cuello, A., Mattei, L. (2012). La Fundación de la Madraza yusufiyya. En Salmerón Escobar, P., Pérez de la Torre, R. (Eds.), Guía breve del Palacio de la Madraza (pp. 33-37). Granada: Universidad de Granada.

(22) Malpica Cuello, A., Mattei, L. (2012). Investigación arqueológica. En Salmerón Escobar, P., Pérez de la Torre, R. (Eds.), Guía breve del Palacio de la Madraza (pp. 77-85). Granada: Universidad de Granada.

(23) Mattei, L. (2008). Estudio de la Madraza de Granada a partir del registro arqueológico y de las metodologías utilizadas en la intervención del 2006. Arqueología y Territorio (5): 181-192.

(24) Mattei, L. (2009). La evolución urbana del entorno de la Madraza Yusufi yya a través de su intervención arqueológica. Revista de arqueología municipal de Silves, Xelb, 9: 493-505.

(25) Almagro Gorbéa, A. (2007). Levantamiento planimétrico del Palacio de la Madraza. En López Guzmán, R., Díez Jorge, M. ${ }^{\text {a }}$ E. (Eds.), La Madraza: pasado, presente y futuro (pp. 323-334). Granada: Universidad de Granada.

(26) Salmerón, P. (2007). Restauración arquitectónica en el Palacio de la Madraza. En López Guzmán, R., Díez Jorge M. a E. (Eds.), La Madraza: pasado, presente y futuro (pp. 161-225). Granada: Universidad de Granada.

(27) Jiménez Domínguez, M. (2007). Dibujos. En López Guzmán, R., Díez Jorge, M. ${ }^{a}$ E. (Eds.), La Madraza: pasado, presente y futuro (pp. 335-341). Granada: Universidad de Granada.

(28) Ramos Molina, J. (2015). Estudio de materiales y técnicas constructivas de elementos singulares en el Palacio de la Madraza. Protocolo de actuación. (Tesis doctoral no publicada). Granada: Universidad de Granada.

(29) García Bueno, A., Hernández Pablos, A., Medina Flórez, V. J. (2007). Estudio previo a la restauración de las yeserías

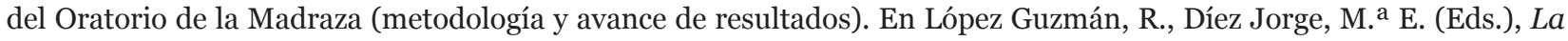
Madraza: pasado, presente y futuro (pp. 277-304). Granada: Universidad de Granada.

(30) García Bueno, A., Medina Flórez, V. J., González Segura, A. (2010). Las yeserías del Oratorio de la Madraza de Yusuf I, Granada. Aportaciones de la documentación gráfica a la determinación de zonas originales y añadidos en el estudio preliminar. Al-Qantara, 31(1): 257-267.

(31) Martín Peinado, B., Albert Arpa, A. (2007). Estudios previos a la intervención sobre la fachada de la Madraza de Granada. En López Guzmán, R., Díez Jorge, M. a E. (Eds.), La Madraza: pasado, presente y futuro (pp. 227-276). Granada: Universidad de Granada.

(32) Romero Pastor, J. (2011). Interaction between historic painting materials. Benefit of applyng sepectrometric techniques and principal component analysis (Tesis doctoral publicada). Granada: Universidad de Granada.

(33) Romero, J., Durán, A., Rodríguez-Navarro, A., Van Grieken, R., Cardel, C. (2011). Compositional and Quantitative Microtextural characterization for historic Paintings by Micro-X-ray Diffraction and Raman Microscopy. Analytical Chemistry, 83(22): 8420-8428, doi: https://dx.doi.org/10.1021/ac201159e.

(34) García Bueno, A., Medina Flórez, V. J. (2004). The Nasrid plasterwork at "qubba Dar al-Manjara l-kubra" in Granada: characterisation of materials and techniques. Journal of Cultural Heritage, 5: 75-89, doi: https://doi.org/10.1016/j. culher.2003.02.002.

(35) García Bueno, A., Medina Flórez, V. J., González Segura, A. (2010). La policromía de las yeserías del oratorio de la Madraza de Yusuf I, Granada. Primeras aportaciones del estudio de materiales para la localización de zonas originales y añadidos. Al-Qantara, 31(1): 245-256.

(36) Golvin, L. (1986, diciembre). Quelques réflexions sur la fondation d'une madrasa à Grenade. 750-1349. En Actas del XII Congreso de la U.E.A.I. (pp. 305-313). Málaga.

(37) Salmerón, P. Restauración del patrimonio histórico. Arquitectura medieval hispanomusulmana. Palacio de la Madraza. Granada. http://www.pedrosalmeron.com/2p2-madraza.

(38) López Lorente, V. (2014). Madraza de Granada. La Madraza nazarí de Yusuf I. http://www.arteguias.com/mezquita/ madrazagranada.htm

(39) NORMAL-1/88. (1990). Alterazione macroscopiche dei materiali lapidei: lessico, 36 pp. CNR-Consiglio Nazionale delle Ricerche. Centri di studio di Milano e Roma sulle cause di deperimento e sui metodi di conservazione delle opere d'arte. Istituto Centrale per il Restauro. 